\section{Original Article}

Check for updates

\section{OPEN ACCESS}

Received: Mar 6, 2019

Revised: Jun 12, 2019

Accepted: Aug 18, 2019

Correspondence to

Roberto Tozzi

Department of Gynaecologic Oncology, Oxford

Cancer Centre, Churchill Oxford University

Hospital, Old Road OX3 7LJ, Oxford, United

Kingdom.

E-mail: roberto.tozzi@obs-gyn.ox.ac.uk

Copyright (c) 2020. Asian Society of Gynecologic Oncology, Korean Society of

Gynecologic Oncology, and Japan Society of

Gynecologic Oncology

This is an Open Access article distributed under the terms of the Creative Commons Attribution Non-Commercial License (https:// creativecommons.org/licenses/by-nc/4.0/) which permits unrestricted non-commercial use, distribution, and reproduction in any medium, provided the original work is properly cited.

ORCID iDs

Roberto Tozzi (iD)

https://orcid.org/0000-0001-6123-1628

Federico Ferrari (D)

https://orcid.org/0000-0001-7065-2432

Joost Nieuwstad (iD

https://orcid.org/0000-0002-9273-6896

Riccardo Garruto Campanile (D)

https://orcid.org/0000-0003-0009-8389

Hooman Soleymani Majd (D)

https://orcid.org/0000-0003-3293-5321

Conflict of Interest

No potential conflict of interest relevant to this article was reported.

\title{
Tozzi classification of diaphragmatic surgery in patients with stage IIIC-IV ovarian cancer based on surgical findings and complexity
}

\author{
Roberto Tozzi (1), Federico Ferrari $(\mathbb{D}$, Joost Nieuwstad (1), \\ Riccardo Garruto Campanile (10, Hooman Soleymani Majd (1)
}

Department of Gynaecologic Oncology, Oxford Cancer Centre, Churchill Oxford University Hospital, Oxford, United Kingdom

\section{ABSTRACT}

Objective: To introduce a systematic classification of diaphragmatic surgery in patients with ovarian cancer based on disease spread and surgical complexity.

Methods: For all consecutive patients who underwent diaphragmatic surgery during VisceralPeritoneal debulking (VPD) in the period 2009-2017, we extracted: initial surgical finding, extent of liver mobilization and type of procedure. Combining these features, we aimed to classify the surgical procedures necessary to tackle different presentation of diaphragmatic disease. We also report histology, intra- and post-operative specific complication rate based on the classification.

Results: A total of 170 patients were included in this study, 110 (64.7\%) had a peritonectomy, while $60(35.3 \%)$ had a full thickness resection with pleurectomy. We identified 3 types of surgical procedures. Type I treated 28 out of 170 patients $(16.5 \%)$ who only had anterior diaphragm disease, needed no liver mobilization, included peritonectomy and had no morbidity recorded. Type II pertained to 105 out of 170 patients $(61.7 \%)$ who had anterior and posterior disease, needed partial and sometimes full liver mobilization, had a mix of peritonectomy and full thickness resection, and experienced $10 \%$ specific morbidity. Type III included 37 out of 170 patients (21.7\%) who needed full mobilization of the liver, always had full thickness resection, and suffered 30\% specific morbidity.

Conclusion: Diaphragmatic surgery can be classified in 3 types. The adoption of this classification can facilitate standardization of the surgery, comparison of data and define the expertise required. Finally, this classification can be a benchmark to establish the training required to treat diaphragmatic disease.

Keywords: Diaphragm; Liver; Peritoneal Neoplasm/Surgery; Peritoneum/Surgery; Classification; Ovarian Cancer

\section{INTRODUCTION}

Ovarian cancer accounts for nearly $4 \%$ of all female cancers. Due to the lack of specific symptoms, 3-quarters of all patients affected by ovarian cancer present with advanced stage disease (International Federation of Gynecology and Obstetrics stage IIIC-IV). Debulking 


\section{Author Contributions}

Conceptualization: T.R.; Data curation: T.R., N.J., C.R.G., S.M.H.; Formal analysis: T.R., F.F.; Investigation: T.R.; Methodology: T.R.; Resources: T.R.; Software: T.R.; Supervision: T.R., F.F.; Validation: T.R., F.F.; Visualization: T.R., F.F.; Writing - original draft: T.R.; Writing review \& editing: T.R., F.F., S.M.H. surgery remains the cornerstone of management for these patients, either as the primary treatment modality or after neo-adjuvant chemotherapy. The residual disease after surgery, irrespective of the timing of the surgery, significantly affects prognosis [1-9]. A complete resection (CR) is associated to the best overall and disease-free survival [5]. In over $70 \%$ of these patients the peritoneum covering the diaphragm and the liver is found with metastatic disease [9]. In $1 / 4$ of the patients the disease involves the diaphragm full thickness [8,9]. The surgical technique can range between a diaphragmatic peritonectomy and a full resection of the peritoneum, muscle and pleura with access to the thorax. Since gynecologic oncologist aiming at a CR of disease are commonly faced with this finding, over the last 10 years surgery of the diaphragm has been incorporated in the debulking procedure. Despite almost 30 years have elapsed since the first report on the surgical resection of diaphragmatic disease in patient with ovarian cancer [6,7], no systematic classification has been proposed on the pattern of diaphragmatic disease and the surgery required. That makes difficult to plan the appropriate surgery, the support needed, provide morbidity figure and compare data. In addition, it is impossible, without a classification, to establish the level of training needed to undertake these procedures. In this study, by reviewing prospectively collected data, we introduce a new classification of diaphragmatic surgery based on the initial extent of disease and on the complexity of the procedure. We also correlate with histology findings and the morbidity rate.

\section{MATERIALS AND METHODS}

Between January 2009 and April 2017, 270 consecutive patients with stage IIIC-IV ovarian cancer were offered Visceral-Peritoneal Debulking (VPD) at 3 institutions under the care of one of the authors (RT): Istituto Clinico Humanitas (ICH, Milan, Italy), Fondazione San Raffaele Giglio (Cefalù, Palermo, Italy), and the Oxford University Hospital (OUH, Oxford, UK). All patients were seen in the Department of Gynaecologic Oncology and discussed in a multi-disciplinary team (MDT) meeting.

They underwent an exploratory laparoscopy (which was always recorded) to assess the feasibility of a CR of all visible tumor according to the criteria outlined in Table 1. Treatment protocols on primary surgery or chemotherapy were previously published [8]. All patients planned for a VPD were electively booked to be admitted in the intensive care unit (ICU). The actual transfer to ICU was decided at the end of the procedure by the anaesthetist mainly based on a successful extubation and cardio-vascular stability. We use a departmental ovarian cancer surgical database to record, monitor and audit surgical data. In this study, a service evaluation project approved by the Oxford University Hospital Trust (ref. 3265), we selected all patients who underwent diaphragmatic surgery in this period. Clinical notes, surgical details, laparoscopic videos, histology and MDT reports were extracted.

Table 1. Inclusion and exclusion criteria for VPD

\begin{tabular}{|c|c|}
\hline Inclusion criteria & Exclusion criteria \\
\hline - Preoperative & - Preoperative \\
\hline - Histology proven or suspected stage IIIC-IV ovarian cancer & - CT scan showing presence of lung metastases, 3 or more liver segments \\
\hline - Performance status (ECOG) $<2$ & involvement and/or disease progression on chemotherapy \\
\hline $\begin{array}{l}\text { - Post chemotherapy patients with stabile disease or response at } 3 \\
\text { or } 6 \text { cycles }\end{array}$ & $\begin{array}{l}\text { - Intraoperative } \\
\text { - Explorative laparoscopy showing diffuse small bowel serosal deposit, porta } \\
\text { hepatis encasement }\end{array}$ \\
\hline
\end{tabular}

CT, computed tomography; ECOG, Eastern Cooperative Oncology Group; VPD, Visceral-Peritoneal debulking. 
From these documents we aimed at discerning: 1) Initial finding of diaphragmatic disease at surgery classified as: only anterior diaphragm (the entire disease was immediately visible by gentle dorsal pressure of the liver), anterior and posterior (disease extending above and behind the liver extending to the bare area and requiring liver mobilization to be entirely visible), anterior, posterior and approaching the hepato-caval ligament (disease extending from the anterior part of the diaphragm all the way to the hepato-caval ligaments with or without compression on the IVC or hepatic veins); 2) Extent of right liver mobilization: none (dorsal pressure of the liver), partial (ventral liver ligaments, i.e., falciform, anterior coronary), full (ventral and dorsal ligaments, including entire coronary, triangular and hepato-caval); 3) Type of procedure performed (peritonectomy vs. full thickness resection with pleurectomy). The aim was to combine these outcomes in order to design a classification merging the disease findings with the surgical procedure. We also extracted the size of the specimen as per histology report, the rate of admission to ICU, rate of intraand post-operative specific morbidity (defined as complications clearly associated to the diaphragmatic surgery, occurring during the operation or within 90 days of the operation).

The surgical technique was partially described before [9]. We hereby report, in steps, the standard surgical procedure consistently performed to tackle diaphragmatic disease (Table 2). In our practice we consider surgical resection the only option and do not perform coagulation, fulguration or ablation. Since the surgery was customized to the extent of disease, not the entire procedure was performed in all patients. Also, the order of the following steps was not always respected, usually seeking the safest possible access to the diaphragm. Before initiating the actual surgery, a proper overview of the extent of disease was undertaken by inspection and palpation. The procedure started by coagulation and cut of the caudal part of the falciform ligament 3-4 cm distal to the out spring from the liver (step 1). This step was undertaken at the beginning of the laparotomy, even before placing the Bookwalter retractor to provide adequate traction on the abdominal wall and avoid inadvertent liver bleeding. The stump of the ligament was used to apply gentle traction on the liver initially and then helped the resection of the actual ligament teres hepatis from the liver insertion which was often site of disease. Another preliminary step was the access to the porta hepatis with encirclement of the hepatic triads by mean of a vessel loop to prepare for a Pringle manoeuvre if it became necessary. The resection continued with the membranous part of the falciform ligament towards the insertion in the diaphragm (step 2). The line of resection of the membranous part of the falciform ligament was caudo-cranial. The resection of the falciform ligament allowed moderate mobility of the superior aspect of the liver and was continued until the

Table 2. Eleven steps of diaphragmatic surgery in patients with stage IIIC-IV ovarian cancer according to Tozzi classification

\begin{tabular}{|c|c|c|c|c|}
\hline Step & Description & Type I & Type II & Type III \\
\hline 1 & Resection of the falciform ligament $3-4 \mathrm{~cm}$ ventral to the out spring from the liver & $\mathrm{R}$ & $\mathrm{R}$ & $\mathrm{R}$ \\
\hline 3 & Resection of anterior part of the right coronary ligament until the suprarenal impression of the liver & & $\mathrm{R}$ & $\mathrm{R}$ \\
\hline 5 & Resection of the dorsal ligament (hepato-caval) until the inset of the hepatic vein & & & $\mathrm{R}$ \\
\hline 6 & Exposure of right and middle hepatic veins $+/$ - encirclement with vessel loop & & & $\mathrm{R}$ \\
\hline 7 & Marking the peritoneal disease below the ribs to start ventral peritonectomy & $\mathrm{R}$ & $\mathrm{R}$ & $\mathrm{R}$ \\
\hline 9 & Incision of the muscle tailored to the extent of the disease & & & $\mathrm{R}$ \\
\hline 10 & $\begin{array}{l}\text { Repair of the defect with or without a mesh, with a Foley catheter and a Valsalva manoeuvre to restore the } \\
\text { negative pressure }\end{array}$ & & & $\mathrm{R}$ \\
\hline 11 & Test to prove the integrity of the diaphragm & & & $\mathrm{R}$ \\
\hline
\end{tabular}

$\mathrm{R}$, required; VPD, Visceral-Peritoneal debulking. 
membranous part divides into the right and left part of the anterior portion of the coronary ligament. Next step (step 3) was the resection of anterior part of the right coronary ligament which was kept under tension by gentle retraction of the renal and duodenal impression until the suprarenal impression was accessed. Thanks to the progressive mobilization of the liver, the dorsal diaphragm was exposed. To fully access the area, the right triangular ligament was resected and the latter part of it, the hepato-renal ligament was also resected (step 4). Finally, in patients whose disease abutted the IVC, the right adrenal gland was exposed; the dorsal ligament of the liver or so called hepato-caval ligament was resected until the inset of the right hepatic vein was exposed (step 5). If the disease was expanding towards the tendon pillars than also the middle hepatic vein was exposed. In patients where the disease was abutting the veins, they were mobilised proximally and distally, freed and, if necessary, encircled with a vessel loop to guarantee vascular control (step 6). During the resection of the dorsal ligament, ligature of accessory veins occurred if necessary. At that stage the liver was fully rotated, the right hepatic vein inset in the cava was exposed. Once the appropriate exposure was achieved, the right upper sub-costal blade of the Bookwalter retractor was removed to mark the exact site of the peritoneal disease and start the ventral peritonectomy from there (step 7). The initial peritoneal incision was made as wide as possible in order to immediately broaden the surface of traction and prepare an en-bloc resection. The retractor was placed again, and the peritoneum pulled gently away from the muscle in a centripetal fashion. Same would be done from the upper part of the paracolic gutter and over the right kidney (step 8) converging towards the tendon. Care would be given if the disease was very close to the vessels especially the IVC which was mobilised laterally and posteriorly away from the peritoneum. When the disease was abutting the IVC or the hepatic veins, namely the right hepatic vein, the vessels were exposed proximally and distally to permit the placement of a vascular clamp if needed. In some cases, a vessel loop was passed behind to apply gentle traction and occlude the vessel in case of injury. The technique for full thickness resection with pleurectomy and repair was described before [9]. We summarise this procedure into 3 additional steps: the incision of the muscle and the overlying pleura (step 9) addressed by the extent of the disease on both sides of the diaphragm; the repair of the defect with or without a mesh, with a Foley catheter and a Valsalva manoeuvre to restore the negative pressure and avoid pneumothorax (step 10); the control of the integrity of the closure by an air test (step 11).

Our post-operative protocol was previously described [9]. Briefly we routinely don't insert thoracic drain irrespective of the type of surgery. We undertake chest $\mathrm{X}$-ray on day $\mathrm{O}$ or once patient is extubated to verify for the presence of pneumothorax. If the latter is found, we only place a chest tube when symptoms require. Intra-operative morbidity events were graded with the CLASSIC score, post-operative with the Clavien-Dindo classification. Data were analyzed using the $\chi^{2}$ test or Fisher's exact test for categorical variables or proportional differences and the Student's t-test for continuous variables. A p-value of 0.05 or $<0.05$ was considered statistically significant.

\section{RESULTS}

In the study period 170 patients underwent diaphragmatic surgery. They build the study group: 93 patients out of $170(54.7 \%)$ underwent primary VPD (group 1) and 77 out of 170 (45.2\%) underwent VPD after platinum-based chemotherapy (group 2). Patient and tumor characteristics are reported in Table 3. Based on the surgical findings, we recorded 28 patients with anterior diaphragmatic disease only, 105 patients with anterior and posterior 
Table 3. Patients and tumor characteristics in group 1 (primary VPD) and group 2 (neo-adjuvant VPD)

\begin{tabular}{lcccc}
\hline Characteristics & Group 1 $(\mathrm{n}=93)$ & Group 2 $(\mathrm{n}=77)$ & Total $(\mathrm{n}=170)$ & $\mathrm{p}$-value (group 1 vs. group 2) \\
\hline Age & $63(52-72)$ & $66(51-79)$ & $65(51-79)$ & - \\
$\begin{array}{l}\text { Previous treatment } \\
\quad\end{array}$ & & & NA & \\
$\quad$ None & $93(54.7)$ & - & & \\
$\quad$ Chemotherapy & - & $77(45.2)$ & & 0.86 \\
$\begin{array}{l}\text { FIGO stage } \\
\quad \text { IIIC }\end{array}$ & $68(73.1)$ & $65(84.4)$ & $133(78.2)$ & 0.65 \\
$\quad$ IV & $25(26.9)$ & $12(15.6)$ & $37(21.8)$ & 0.90 \\
Histology type & & & & 0.91 \\
$\quad$ HGSC & $80(86.2)$ & $66(85.7)$ & $146(85.8)$ & \\
$\quad$ Others & $13(13.8)$ & $11(14.2)$ & $24(14.2)$ & 0.88 \\
Tumor grade & & & & 0.64 \\
$\quad$ G1 & $7(7.5)$ & $5(6.5)$ & $12(12.7)$ & 0.72 \\
$\quad$ G2 & $12(12.9)$ & $3(3.9)$ & $15(5.3)$ & \\
$\quad$ G3 & $74(79.5)$ & $69(89.6)$ & $143(82)$ & \\
\hline
\end{tabular}

Data are presented as number (range) or number (rate).

FIGO, International Federation of Gynecology and Obstetrics; HGSC, High Grade Serous Cancer; NA, not available; VPD, Visceral-Peritoneal debulking.

disease and 37 patients with disease extending from the anterior diaphragm to the proximity of the hepatic vessels. Based on the liver mobilization, 28 patients needed none, 105 patients partial and 37 patients needed a full mobilization with resection of the hepato-caval ligament. Out of the overall 170 patients, $110(64.3 \%)$ had a peritonectomy alone, 60 (35.7\%) had a peritonectomy and full thickness resection with pleurectomy. One hundred seventeen patients out of 170 went to intensive therapy unit (ITU) after the procedure. We recorded overall 22 morbidity events in 21 patients out of 170 (11.7\%): 2 intra-operative complications (1 injury of the supra-hepatic IVC, 1 of the right hepatic vein), 2 shortly postoperative (both events were consistent with liver prolapse in the chest secondary to suture dehiscence) and 18 lately postoperative ( 13 pleural effusions and 5 pneumothorax). The morbidity events were graded as G3 in 4 patients and G2 in 16 patients. Based on the distribution of these 3 outcomes (initial findings, liver mobilization, surgical procedure) we describe 3 types of diaphragmatic surgery (Table 4) for which we also report size specimen and morbidity.

Type I surgery (Fig. 1) was performed in 28 out of 170 patients (16.5\%). They had disease limited to the anterior aspect of the diaphragm, needed no liver mobilization, underwent peritonectomy only and had no morbidity recorded. This procedure included steps 1-2, 7-8. Only 8 patients out of 28 (28.5\%) went to ICU post-operatively and stayed for 1 day (range $1-2)$. These 8 patients went to ITU due to coexisting morbidity unrelated to the surgery. The mean size of the resected specimen was $11 \mathrm{~cm}$ (range 5-18 cm).

Table 4. Tozzi classification of diaphragmatic surgery based on disease findings, liver mobilization and procedure with size of specimen and morbidity rate

\begin{tabular}{|c|c|c|c|c|}
\hline Characteristics & Type I $(n=28)$ & Type II $(n=105)$ & Type III $(n=37)$ & $p$-value \\
\hline Disease findings & Anterior & Anterior/posterior & $\begin{array}{l}\text { Anterior/posterior/abutting } \\
\text { the hepatic veins }\end{array}$ & - \\
\hline Liver mobilization & None & $\begin{array}{l}\text { Partial without } \\
\text { dorsal ligament }\end{array}$ & $\begin{array}{l}\text { Full including } \\
\text { dorsal ligament }\end{array}$ & - \\
\hline Procedure & Peritonectomy & Peritonectomy/resection & Resection & - \\
\hline $\begin{array}{l}\text { Maximum size of the } \\
\text { specimen in } \mathrm{cm}\end{array}$ & $11(5-18)$ & $19(12-29)$ & $23(15-36)$ & $\begin{array}{l}0.05 \text { (I vs. II) } \\
0.03 \text { (I vs. III) } \\
0.52 \text { (II vs. III) }\end{array}$ \\
\hline Morbidity & - & $9(9.5)$ & $11(29.7)$ & 0.02 (II vs. III) \\
\hline
\end{tabular}

Data are presented as number (range) or number (rate). 


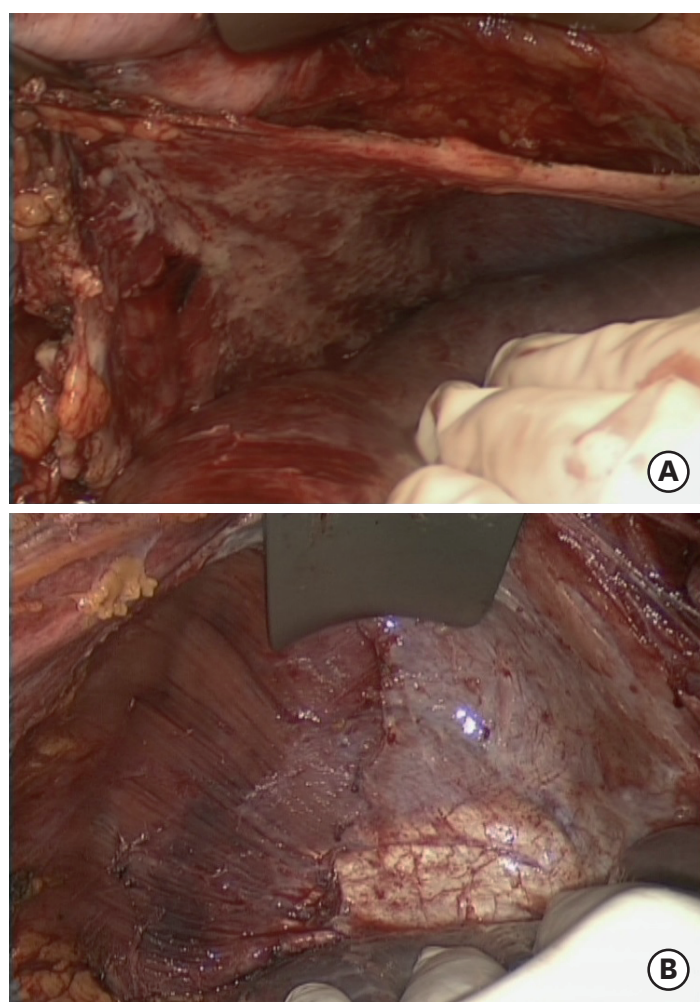

Fig. 1. Type I diaphragmatic surgery according to Tozzi classification, initial finding (A) and final outcome (B).

Type II surgery (Fig. 2 and Supplementary Fig. 1) pertained to 105 out of 170 patients (61.7\%) who had anterior and posterior disease, needed partial liver mobilization without dorsal ligaments, had a combination of peritonectomy (68 patients) and full thickness resection (37 patients). Morbidity affected 10 patients out $105(9.5 \%)$ and included 6 cases of postoperative pleural effusion, none of which required drainage and 3 events of pneumothorax. All these patients had G1 events except 1 who had a G3 event requiring chest tube for pneumothorax. In addition, one patient experienced a post-operative liver herniation into the chest. The latter was secondary to the dehiscence of the diaphragmatic suture following a full thickness resection. It was repaired through an abdominal approach with re opening of the supra umbilical part of the laparotomy. The liver was manually pulled back in the abdomen and the diaphragm sutured with 0 PDS stitch with the aid of a Prolene mesh to reinforce the strength of the repair. The patient made a quick and full recovery and was discharged 8 days after the second surgery, 11 after the first one. Type II procedure always included steps 1 to $4,7-8$. In this group, 80 patients out $105(76.1 \%)$ needed post-operative ICU care for a median 1.2 days (range 1-2). The mean size of the specimen resected was $19 \mathrm{~cm}$ (range 12-29 cm).

Type III surgery (Supplementary Figs. 2 and $\mathbf{3}$ ) was performed in 37 out of 170 patients $(21.7 \%)$, with disease from the anterior to the posterior diaphragm and to hepatic vessels. All patients needed full liver mobilization with resection of the dorsal/hepato-caval ligament of the liver and had full thickness resection. Complications were recorded in 11 patients out of $37(29.7 \%)$ and included: 7 cases of pleural effusion, 1 of pneumothorax, 1 of liver prolapsed in the chest, 2 intra-operative vascular accidents. The postoperative events were graded as G1 in 7 patients and G3 in the other 3 patients. The last 3 events are reported in more details. The patient with liver herniation in the chest had a full dehiscence of the diaphragmatic 

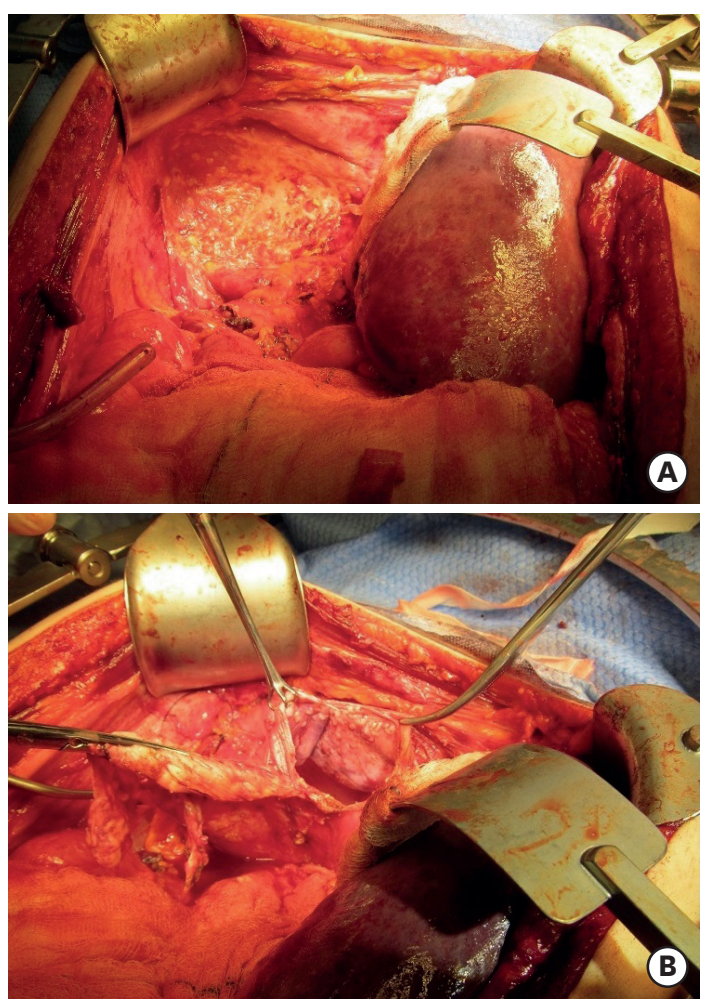

Fig. 2. Type II diaphragmatic surgery according to Tozzi classification, initial finding (A) and open diaphragm (B).

suture performed to repair the diaphragm after a full thickness resection. In this patient a cardio-thoracic surgeon decided to undertake a thoracotomy and repair the diaphragm from the chest. The patient remained intubated in ICU for 8 days, was eventually extubated and recovered well. She was discharged 28 days from the first surgery and 25 days from the second surgery. Two vascular accidents occurred: 1 injury of the anterior wall of the suprahepatic IVC and 1 injury of the right aspect of the right hepatic vein. Both vascular injuries occurred in the initial part of these series. The first patient had disease stuck between the anterior aspect of the right coronary ligament and the IVC. After careful dissection of the ligaments, it looked like a dissection plane existed between the IVC and the most proximal peritoneal disease. During this phase a up to $5 \mathrm{~mm}$ lesion of the IVC was caused. Since the exact source of bleeding could not be immediately seen, the haemorrhage resulted in an overall $300 \mathrm{~mL}$ blood loss and was stopped with mechanical compression. Eventually the injury was visualised and exposed. Due to the challenging angle and the relative lack of liver mobility because of the disease, a vascular clamp could not be placed. Therefore a 6-0 Prolene running suture was used applying some traction to limit the bleeding. The suture was successful, and the surgery was continued to a CR. The second patient had a full liver mobilization and exposure of the right hepatic vein. It was clear that the disease was stuck to the inset of the right hepatic vein into the IVC. Therefore, both the IVC and the right hepatic vein were exposed, and the right hepatic vein was encircled with a vessel loop. Previously the porta hepatis had been isolated and prepared for a Pringle manoeuvre as per protocol. When dissecting the peritoneum form the right hepatic vein a small tear occurred in the right aspect of the vein. Thanks to the extensive preparation the vein was pulled with the vessel loop proximally to the injury, the area of the right hepatic inset in the IVC was occluded with a vascular clamp and the Pringle manoeuvre was applied. Under these circumstances, 
the blood loss was negligible, and it was extremely convenient to repair the hole in the right hepatic vein with 5-0 Prolene. The suture was successful, and the procedure ended with a CR. Type III procedure included all 11 steps in all 37 patients. They all went to ICU postoperatively and remained for a median 1.8 days (range $0-18$ ). The mean size of the resected specimen was $23 \mathrm{~cm}$ (range 15-36 cm).

Supplementary Table 1 is a detailed summary of the intra- and post-operative morbidity with type, grade and rate of the complications.

\section{DISCUSSION}

For over 3 decades, the use of diaphragmatic surgery has been advocated in the debulking of patients with advanced ovarian cancer. Since 2002, over forty studies have been published reporting on various techniques, clinical and survival outcomes [10-18]. Between 50\% and $90 \%$ of patients with stage IIIC-IV have diaphragmatic involvement. In these patients a CR would not be achieved without diaphragmatic surgery. Beyond the CR, some studies have associated a survival benefit to diaphragmatic surgery [19]. Despite a noticeable number of reports, surprisingly, no standardized classification existed so far on the types of diaphragmatic surgery. When adding a new technique to a standardized operation, it is important that the outcomes are tested and monitored. However, if no definition of the surgery and no consistent terminology exist, there is a substantial risk that different disease findings and operations of variable complexity are pulled together under the denomination diaphragmatic surgery. The classification we designed takes in account all these valuable outcomes and combines them together. It provides information to assist MDTs in addressing the most appropriate management and gynecologic oncologists to appropriately consent patients and prepare for the surgery. We summarise in Supplementary Table 2 the requirements for each type of surgery.

Type I operations are relatively straight forward. They do not add specific morbidity to the debulking surgery and are usually associated to less complex operations. The surgeon needs to have expertise in peritonectomy and be aware of how to deal with accidental opening of the pleura. That includes the technique to restore negative pressure or to place a chest drain and to repair the diaphragm. However, in our study, no liver mobilization was needed nor was a full thickness resection. Therefore, we believe that there is no need for a specific training to undertake this surgery. Patients should be informed of the minimal risk of accidental opening of the chest and the rare event of pneumothorax. This category of patients is unlikely to need ITU.

All gynecologic oncologists aiming at CR should be familial with type II operation because is the most commonly performed surgery. The findings on the diaphragm are extensive; do often require a full thickness resection and always a liver mobilization. The surgeon embarking on this type of surgery needs to have full knowledge of the liver mobilization technique and the anatomy of the area including the position of the vessels. Although it was only necessary in one patient, the Pringle manoeuvre was essential in preventing a major haemorrhage. We believe that a routine exposure of the hepatic hilum with elective placement of the vessel loop is a safety measure that contributes to the low morbidity. In addition, surgeons performing type II operations should be acquainted with the resection and repair of the diaphragm. Also, they should be familiar with the technique of restoring 
negative pressure in the pleural cavity by use of Foley catheter to apply suction combined with manual maximal ventilation to expand the lungs. The use of such technique contributed in our series to a surprising low rate of pneumothorax, 5 patients out of $60(8.3 \%)$. This is a valuable outcome since pneumothorax can significantly complicate the recovery and requires often the placement of a thoracic drain. In terms of preparation, patients undergoing a type II surgery should be informed of the specific morbidity including the possibility of a chest drain to deal with pneumothorax or pleural effusion and, in rare cases, of a prolonged ITU stay. Since these patients were likely to need ITU stay, that should be planned for. We electively booked ITU stay for patients undergoing VPD but it was ultimately the anaesthetist decision to use the bed. It is very difficult to weigh the impact of the diaphragmatic surgery on the decision but, irrespective, it is fair to say that more complex diaphragmatic surgery was associated to more complex debulking surgery.

Type III operations are the most complex procedures and are associated to the highest risk of intra- and post-operative morbidity. Detailed knowledge of the hepatic vascular anatomy, specifically the dorsal or hepato-caval ligament, is essential, including recognition of the small accessory veins draining in the hepatic part of the IVC. Likewise, the technique to achieve vascular control with isolation of the hepatic veins and the IVC is mandatory. Finally, the diaphragmatic part of the surgery was always consistent with a full thickness resection. Therefore, type III surgery requires expertise in all the steps previously mentioned for the reconstruction of the diaphragm and, additionally, with the use of mesh. Most of these surgical steps of type III are not part of the usual training of gynecologic oncologists. Should the expertise for full liver mobilization and diaphragmatic resection not be independently available, patients should be referred to centres with expertise in ultra-radical debulking or the aid of hepato-biliary surgeons should be sought. Although it is very difficult to make recommendations based on a retrospective analysis, we note that all patients in our study proceeded to ITU after the type III surgery. In these patients, therefore, we continue to plan for ITU if this procedure is anticipated. Patients should be made aware, at time of consent, of the specific morbidity rate. In our personal experience we found rather difficult to differentiate, based on a computed tomography (CT) scan, between patients requiring type II and type III operations. By integrating the information of the CT scan with an exploratory laparoscopy, we could accurately anticipate the exact extent of diaphragmatic disease and prepare for the correct surgery. The actual limit of this approach is the assessment of the pleural side of the diaphragm. We do not routinely undertake a thoracoscopy as some studies suggest [20] because we believe that if undertaking diaphragmatic surgery, the team should be able to deal with a full thickness resection. However, if the latter poses a challenge, a combination of an exploratory laparoscopy and thoracoscopy may well be more accurate and provide additional information on both sides of the diaphragm. To help predict the type of diaphragmatic surgery, we verified if the use of neo-adjuvant chemotherapy could affect the surgery. Ninety-three patients in group 1 (primary VPD) and the 77 patients in group 2 (neo-adjuvant VPD) were evenly distributed in the 3 types of diaphragmatic surgery and no statistically significant difference could be identified. We expected these results, because, in terms of surgical complexity, the usually higher tumor load at primary surgery is compensated by the fibrosis and retraction of the tissues caused by neo-adjuvant chemotherapy. We also tried to identify risk factors for diaphragmatic morbidity but failed to. None of the following factors was significantly associated to morbidity: low albumin $(<18$ $\mathrm{UI} / \mathrm{L})$, liver mobilization and type III surgery. 
In conclusion, while any surgeon tackling diaphragmatic disease should have the specialised expertise, there are different levels of complexity and of morbidity associated to different surgical findings. Although several surgeons can be familial with a type II and some degree of liver mobilization, few will be acquainted with the full mobilization of the hepatocaval ligament which presents its own specific complexity. This study introduces a new classification of diaphragmatic surgery combining the surgical findings with the surgical technique and the morbidity associated. It provides clear indications on the necessary surgical steps, the type of training required, the preoperative preparation demanded and the expectable morbidity of the procedures. It can be prospectively used to provide the appropriate surgical expertise and anticipate severe morbidity (such as vascular injury), therefore improve patients' care.

\section{SUPPLEMENTARY MATERIALS}

\section{Supplementary Table 1}

Procedure specific morbidity by type of diaphragmatic surgery according to Tozzi classification

Click here to view

\section{Supplementary Table 2}

Pre-, intra- and postoperative requirements for diaphragmatic surgery according to Tozzi classification

Click here to view

\section{Supplementary Fig. 1}

Type II diaphragmatic surgery according to Tozzi classification, pleural cavity (A) and final outcome (B).

Click here to view

Supplementary Fig. 2

Type III diaphragmatic surgery according to Tozzi classification, initial finding.

Click here to view

Supplementary Fig. 3

Type III diaphragmatic surgery according to Tozzi classification, final outcome.

Click here to view

\section{REFERENCES}

1. Vergote I, Tropé CG, Amant F, Kristensen GB, Ehlen T, Johnson N, et al. Neoadjuvant chemotherapy or primary surgery in stage IIIC or IV ovarian cancer. N Engl J Med 2010;363:943-53.

PUBMED | CROSSREF 
2. Chi DS, Liao JB, Leon LF, Venkatraman ES, Hensley ML, Bhaskaran D, et al. Identification of prognostic factors in advanced epithelial ovarian carcinoma. Gynecol Oncol 2001;82:532-7. PUBMED | CROSSREF

3. Winter WE 3rd, Maxwell GL, Tian C, Carlson JW, Ozols RF, Rose PG, et al. Prognostic factors for stage III epithelial ovarian cancer: a Gynecologic Oncology Group Study. J Clin Oncol 2007;25:3621-7. PUBMED | CROSSREF

4. Bristow RE, Tomacruz RS, Armstrong DK, Trimble EL, Montz FJ. Survival effect of maximal cytoreductive surgery for advanced ovarian carcinoma during the platinum era: a meta-analysis. J Clin Oncol 2002;20:1248-59. PUBMED | CROSSREF

5. Chi DS, Eisenhauer EL, Lang J, Huh J, Haddad L, Abu-Rustum NR, et al. What is the optimal goal of primary cytoreductive surgery for bulky stage IIIC epithelial ovarian carcinoma (EOC)? Gynecol Oncol 2006;103:559-64. PUBMED | CROSSREF

6. Deppe G, Malviya VK, Boike G, Hampton A. Surgical approach to diaphragmatic metastases from ovarian cancer. Gynecol Oncol 1986;24:258-60. PUBMED | CROSSREF

7. Montz FJ, Schlaerth JB, Berek JS. Resection of diaphragmatic peritoneum and muscle: role in cytoreductive surgery for ovarian cancer. Gynecol Oncol 1989;35:338-40. PUBMED | CROSSREF

8. Tozzi R, Giannice R, Cianci S, Tardino S, Campanile RG, Gubbala K, et al. Neo-adjuvant chemotherapy does not increase the rate of complete resection and does not significantly reduce the morbidity of Visceral-Peritoneal Debulking (VPD) in patients with stage IIIC-IV ovarian cancer. Gynecol Oncol 2015;138:252-8. PUBMED | CROSSREF

9. Soleymani Majd H, Ferrari F, Manek S, Gubbala K, Campanile RG, Hardern K, et al. Diaphragmatic peritonectomy vs. full thickness resection with pleurectomy during Visceral-Peritoneal Debulking (VPD) in 100 consecutive patients with stage IIIC-IV ovarian cancer: a surgical-histological analysis. Gynecol Oncol 2016;140:430-5. PUBMED | CROSSREF

10. Tsolakidis D, Amant F, Leunen K, Cadron I, Neven P, Vergote I. Comparison of diaphragmatic surgery at primary or interval debulking in advanced ovarian carcinoma: an analysis of 163 patients. Eur J Cancer 2011;47:191-8. PUBMED | CROSSREF

11. Einenkel J, Ott R, Handzel R, Braumann UD, Horn LC. Characteristics and management of diaphragm involvement in patients with primary advanced-stage ovarian, fallopian tube, or peritoneal cancer. Int J Gynecol Cancer 2009;19:1288-97. PUBMED | CROSSREF

12. Fanfani F, Fagotti A, Gallotta V, Ercoli A, Pacelli F, Costantini B, et al. Upper abdominal surgery in advanced and recurrent ovarian cancer: role of diaphragmatic surgery. Gynecol Oncol 2010;116:497-501. PUBMED | CROSSREF

13. Chéreau E, Ballester M, Selle F, Cortez A, Pomel C, Darai E, et al. Pulmonary morbidity of diaphragmatic surgery for stage III/IV ovarian cancer. BJOG 2009;116:1062-8. PUBMED | CROSSREF

14. Eisenhauer EL, D'Angelica MI, Abu-Rustum NR, Sonoda Y, Jarnagin WR, Barakat RR, et al. Incidence and management of pleural effusions after diaphragm peritonectomy or resection for advanced mullerian cancer. Gynecol Oncol 2006;103:871-7. PUBMED | CROSSREF

15. Chi DS, Abu-Rustum NR, Sonoda Y, Chen SW, Flores RM, Downey R, et al. The benefit of video-assisted thoracoscopic surgery before planned abdominal exploration in patients with suspected advanced ovarian cancer and moderate to large pleural effusions. Gynecol Oncol 2004;94:307-11. PUBMED | CROSSREF

16. Eisenkop SM, Spirtos NM. What are the current surgical objectives, strategies, and technical capabilities of gynecologic oncologists treating advanced epithelial ovarian cancer? Gynecol Oncol 2001;82:489-97. PUBMED | CROSSREF

17. Chéreau E, Rouzier R, Gouy S, Ferron G, Narducci F, Bergzoll C, et al. Morbidity of diaphragmatic surgery for advanced ovarian cancer: retrospective study of 148 cases. Eur J Surg Oncol 2011;37:175-80. PUBMED | CROSSREF

18. Dowdy SC, Loewen RT, Aletti G, Feitoza SS, Cliby W. Assessment of outcomes and morbidity following diaphragmatic peritonectomy for women with ovarian carcinoma. Gynecol Oncol 2008;109:303-7. PUBMED | CROSSREF 
19. Aletti GD, Dowdy SC, Podratz KC, Cliby WA. Surgical treatment of diaphragm disease correlates with improved survival in optimally debulked advanced stage ovarian cancer. Gynecol Oncol 2006;100:283-7. PUBMED | CROSSREF

20. Diaz JP, Abu-Rustum NR, Sonoda Y, Downey RJ, Park BJ, Flores RM, et al. Video-assisted thoracic surgery (VATS) evaluation of pleural effusions in patients with newly diagnosed advanced ovarian carcinoma can influence the primary management choice for these patients. Gynecol Oncol 2010;116:483-8.

PUBMED | CROSSREF 